\title{
Topological correlations in soap froths
}

\author{
K. Y. Szeto, ${ }^{*}$ T. Aste, ${ }^{\dagger}$ and W. Y. Tam \\ Department of Physics, The Hong Kong University of Science and Technology, Clear Water Bay, Kowloon, Hong Kong
}

(Received 18 February 1998)

\begin{abstract}
Correlation in two-dimensional soap froth is analyzed with the introduction of two point correlators and topological distance. Cells with an equal number of sides repel (with linear correlation) while cells with a different number of sides attract (with nonbilinear correlation) for nearest neighbors, which cannot be explained by the maximum entropy argument. Also, the analysis indicates that froth is correlated up to the third shell neighbors at least. [S1063-651X(98)07108-6]
\end{abstract}

PACS number(s): 82.70.Rr, 02.50.-r, 05.70.Ln

Two-dimensional cellular structures are space-filling disordered partitions of space by cells that are irregular polygons and often appear in nature as in metal grains, biological tissues, and common soap froths [1]. These cellular patterns are usually trivalent (three edges meet at a vertex) due to topological stability. A generic term for these networks is "froth," since the soap-froth is the archetype of such structures. Previous theoretical studies on froth are based on mean field, with minimal consideration on the correlation between cells $[2,3]$. This is understandable as any theoretical description taking correlation effects into account is much more difficult, and the correlation in froth is assumed to be completely described by the Aboav-Weaire law [4-6]. Thus, most of the approaches on froths make some kind of independent bubble approximation, and neighbors are described by a mean field [2] or as random neighbors [3]. It is our aim to report the experimental observations of the correlations beyond nearest neighbors. We find that there are long-range correlations, and that any reasonable description respecting the correlation effects on froth must go at least to the third shell neighbors. Furthermore, these correlation effects are not describable by the maximum entropy arguments $[7,8]$, as the predicted bilinearity for the correlators is only a rather crude approximation to the data. The data therefore suggest that there must be a new theory describing real soap froth, with full consideration of the energies and the correlations.

In froths, a cell can be characterized topologically by its number of sides. Froths are disordered systems and a topological, statistical description of their structure is given by the correlation function. To extract the relevant structural properties from a disordered system like a froth is a very difficult task. Indeed, the absence of symmetries and periodicities requires a priori the knowledge of the information about all the cells in the system. A simple and useful approach is to analyze the froth as structured in concentric layers of cells at the same topological distance $j$ from a given central cell [9-12] (where the topological distance between two cells is the minimum number of edges that a path must cross to go from one cell to the other [10-12]). An interest-

\footnotetext{
*Electronic address: phszeto@usthk.ust.hk

${ }^{\dagger}$ Present address: Equipe de Physique Statistique, LUDFC, Université Louis Pasteur, 3 rue de l'Université, 67084 Strasbourg France. Electronic address: tomaso@1dfc.u-strasbg.fr
}

ing and meaningful quantity in this analysis by concentric layers is, for instance, the number of cells in a given layer $\left[K_{j}(n)\right.$, with $n$ the number of sides of the central cell] [11]. Here we focus on the correlation function. The two-cell topological correlation function $\left[C_{j}(n, m)\right]$ is the probability of finding a cell with $m$ sides at a distance $j$ from a cell with $n$ sides. The two-cell topological correlation function is equal to the total number $N_{j}(n, m)$ of couples of cells with, respectively, $n$ and $m$ sides that are at a relative distance $j$, divided by the number of all couples of cells at a relative distance $j$,

$$
C_{j}(n, m)=\frac{N_{j}(n, m)}{\sum_{n, m=3}^{\infty} N_{j}(n, m)} .
$$

We consider the $n$ - and $m$-sided cells as distinguishable. Therefore, each couple is counted twice and $N_{j}(n, m)$ and $C_{j}(n, m)$ are symmetric in $n, m$ [i.e., $\left.C_{j}(n, m)=C_{j}(m, n)\right]$.

By definition, in uncorrelated systems the correlation function must factorize: $C_{j}(n, m)=s_{j}(n) s_{j}(m)$. The normalization and the symmetry of $C_{j}(n, m)$ imply $\Sigma_{m} s_{j}(m)=1$, consequently $s_{j}(n)=\Sigma_{m} C_{j}(n, m)$. Now, we calculate this quantity and we interpret it in terms of the properties of the layers. Consider two cells with $n$ and $m$ sides that are at a relative topological distance $j$. Such a pair, connected by a path of length $j$, can be seen as a "string" with $n$ - and $m$-sided terminations. Clearly the number of these strings in the cellular system is $N_{j}(n, m)$ (where each string is counted twice since the terminations are distinguishable cells). The number of strings of length $j$ with a termination in a cell with $n$ sides and the other termination free is given by $\Sigma_{m} N_{j}(n, m)$. The same quantity is equal to the number of $n$-sided cells in the system $[N(n)]$ multiplied by the average number of strings of length $j$ which terminate in one $n$-sided cell $\left[K_{j}(n)\right]$. Therefore, $\sum_{m=3}^{\infty} N_{j}(n, m)=N(n) K_{j}(n)$. Finally, the total number of strings of length $j$ is equal to the total number of cells $\left(N_{T}\right)$ multiplied by the average number of strings of length $j$ that terminate in any given cell $\left(\left\langle K_{j}\right\rangle\right)$, giving

$$
\begin{aligned}
\sum_{n, m=3}^{\infty} N_{j}(n, m) & =\sum_{n=3}^{\infty} N(n) K_{j}(n) \\
& =N_{T} \sum_{n=3}^{\infty} \frac{N(n)}{N_{T}} K_{j}(n)=N_{T}\left\langle K_{j}\right\rangle,
\end{aligned}
$$



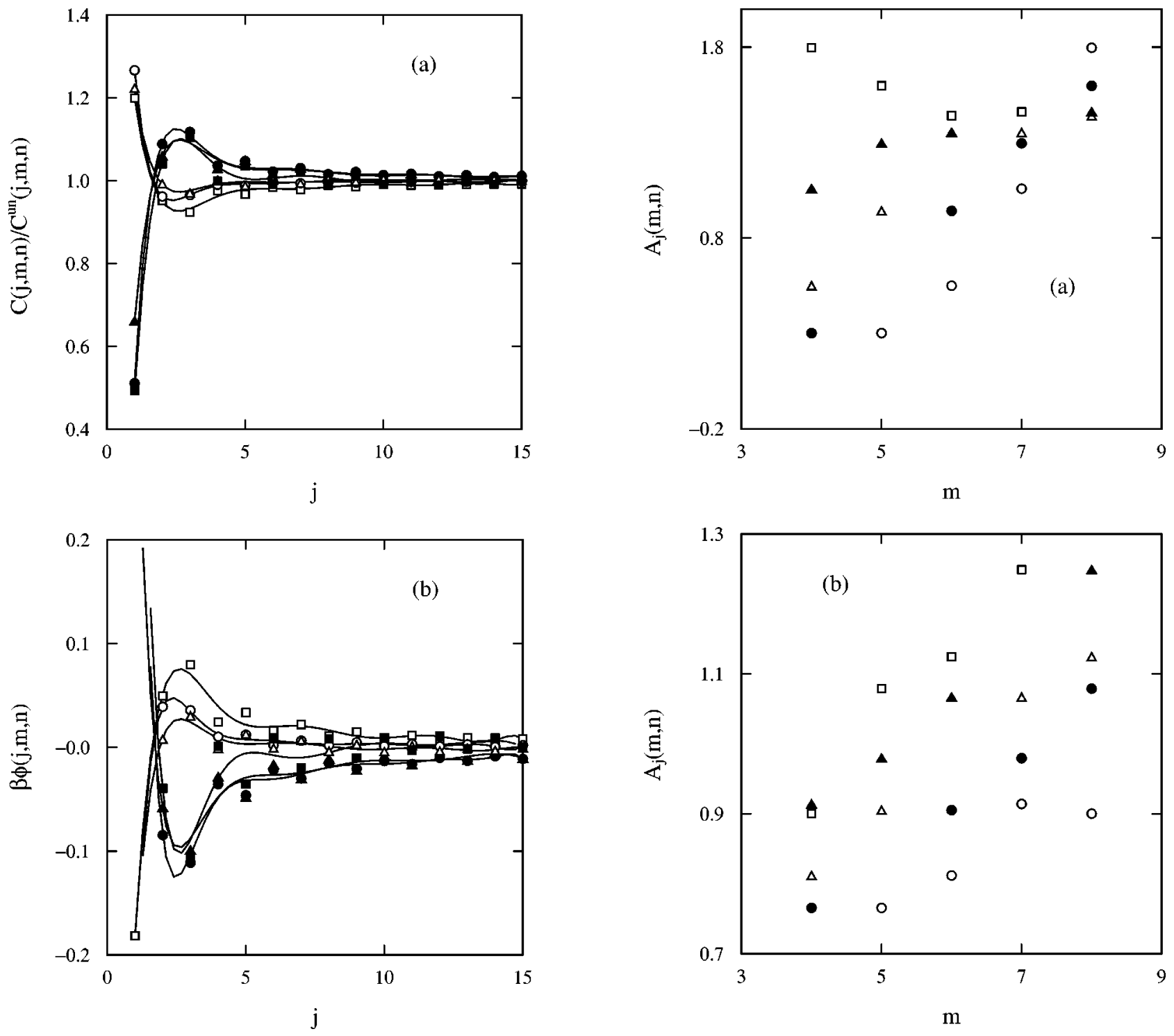

FIG. 1. (a) Relative correlation $C_{j}(n, m) / C_{j}^{u n}(n, m)$ and (b) effective potential $\varphi_{j}(n, m)$ vs topological distance $j$ for couples of soap froth cells with $n$ and $m$ sides, and $(n, m)=(5,5)$, solid circle; $(n, m)=(6,5)$, open square; $(n, m)=(6,6)$, solid triangle; $(n, m)$ $=(7,5)$, open circle; $(n, m)=(7,6)$, open triangle; $(n, m)=(7,7)$, solid square. The solid lines are guides to the eyes.

where $\langle(\cdots)\rangle=\Sigma_{n} p(n)(\cdots)$, with $p(n)=N(n) / N_{T}$ being the probability of an $n$-sided cell in the whole froth. The probability to find a string of length $j$ with a termination in a cell with $n$ sides is consequently given by the ratio

$$
\begin{aligned}
s_{j}(n) & =\frac{\sum_{m=3}^{\infty} N_{j}(n, m)}{\sum_{n, m=3}^{\infty} N_{j}(n, m)} \\
& =\sum_{m=3}^{\infty} C_{j}(n, m)=\frac{N(n) K_{j}(n)}{N_{T}\left\langle K_{j}\right\rangle}=p(n) \frac{K_{j}(n)}{\left\langle K_{j}\right\rangle} .
\end{aligned}
$$

By following this point of view, the correlation function $C_{j}(n, m)$ can be interpreted as the probability of having a string of length $j$ with terminations in two cells with $n$ and $m$

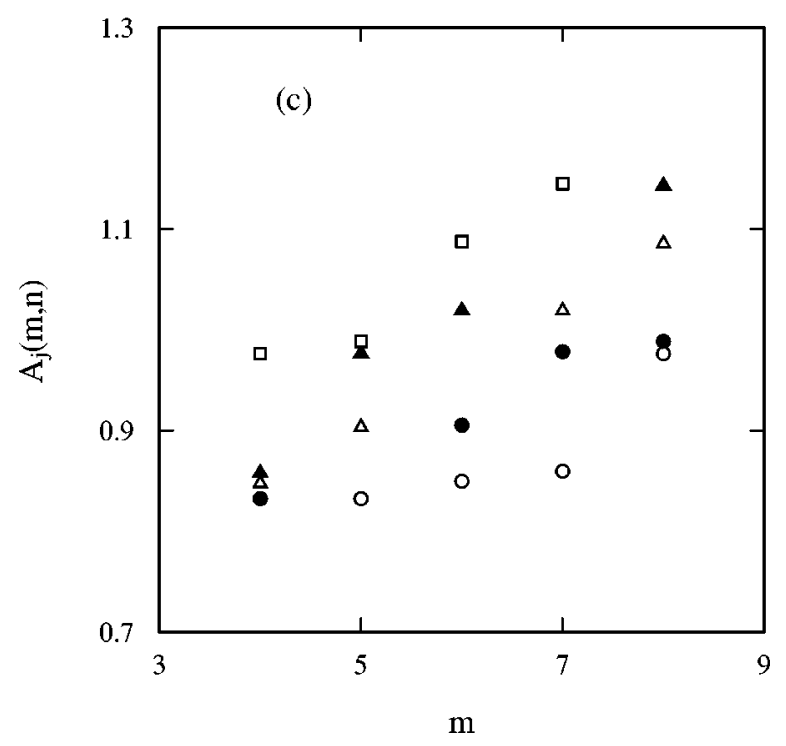

FIG. 2. Correlator $A_{j}(n, m)$ vs $m$ for fixed $n \neq m$, and (a) $j=1$, (b) $j=2$, and (c) $j=3$. Here $n=4$, open circle; $n=5$, solid circle; $n=6$, open triangle; $n=7$, solid triangle; $n=8$, open square. 


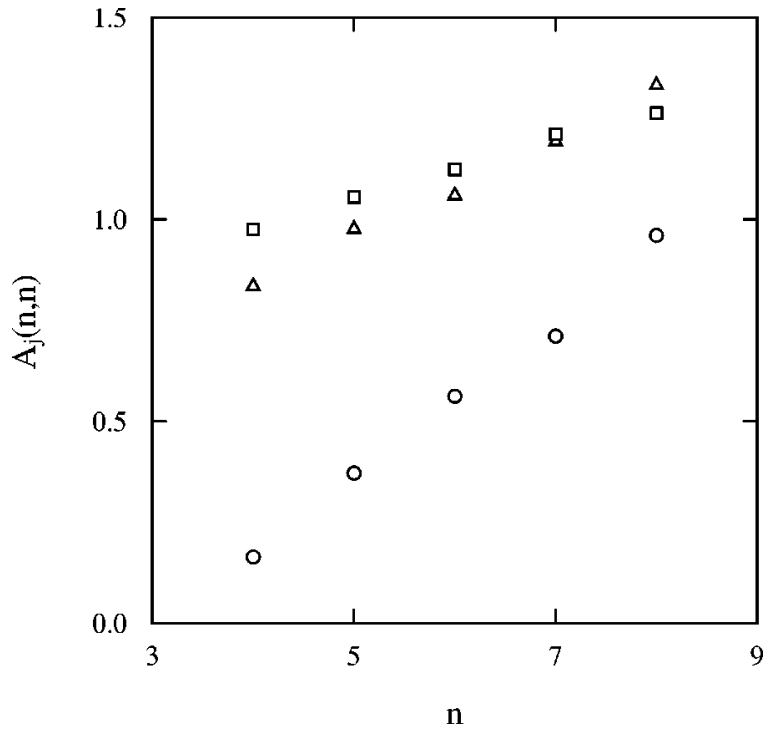

FIG. 3. Correlator $A_{j}(n, n)$ vs $n$ for fixed $j$; (a) $j=1$, open circle; (b) $j=2$, open triangle; (c) $j=3$, open square.

sides, respectively. In an uncorrelated system this conditional probability must be the product of the two string probabilities,

$$
C_{j}^{\mathrm{un}}(n, m)=s_{j}(n) s_{j}(m)=\frac{K_{j}(n) K_{j}(m)}{\left\langle K_{j}\right\rangle^{2}} p(n) p(m) .
$$

Even when the system is uncorrelated, taking two cells at distant $j$, the probability to have one cell with $m$ sides and the other cell with $n$ sides is not given by the simple product of the probabilities of finding independently an $n$ - and an $m$ sided cell $[p(n) p(m)]$. The factor $K_{j}(n) K_{j}(m) /\left\langle K_{j}\right\rangle^{2}$ in Eq. (4) indicates that a cell in a froth (even in an uncorrelated one) cannot be topologically independent of its neighbors. Indeed, in froths, a single isolated cell does not exist. The cell, its number of sides, and the number of neighbors at any distance $j$ constitute a unique system. [Note that, for $j=1$, the function $C_{1}(n, m) / C_{1}^{u n}(n, m)-1=\beta_{n, m}$ is the topological short-range order coefficient introduced by Le Caër et al. [13].]

The structure of a froth can be studied in term of an effective "potential" between different cells. These potentials can correspond to a real dynamical interaction between coupled cells during the process of formation and evolution of the system, or they can simply indicate the degrees of affinities of two cells to stay at a given relative distance. Attractive interactions are associated to couples of cells that appear in the froth with a higher probability than in the uncorrelated case, while negative interactions correspond to the opposite case. Without any loss in generality one can define the correlation function of the form

$$
C_{j}(n, m)=C_{j}^{\mathrm{un}}(n, m) \exp \left(-\beta \varphi_{j}(n, m)\right) .
$$

Here $\varphi_{j}(n, m)$ is the interaction effective potential between two cells with $n$ and $m$ sides at topological distance $j$, and $\beta$ is the inverse temperature. This potential is zero in uncorrelated systems, negative when two cells attract, and positive when they repel.

We have analyzed data for soap froths prepared at different times, all in the scaling regime $[14,15]$. In Fig. 1, we show the variation of $C_{j}(n, m) / C_{j}^{\mathrm{un}}(n, m)$ [Fig. 1(a)] and $\varphi_{j}(n, m)$ [Fig. 1(b)] vs topological distance $j$. These correlations and potentials are the same for a sample that is $6 \mathrm{~h}$ in the scaling regime as another sample that is $14 \mathrm{~h}$ in the scaling regime. Other times have also been checked and $C_{j}(n, m) / C_{j}^{\mathrm{un}}(n, m)$ and $\varphi_{j}(n, m)$ are time independent within experimental errors. (The error bar is within the size of the symbol). From Fig. 1(a), it is clear that soap froth is strongly correlated at least up to the third neighbors. Cells with an equal number of sides [e.g., $(n, m)=(5,5),(6,6)$, $(7,7)]$ have a lower probability to be first neighbors than in the uncorrelated case and the contrary is for cells with a different number of sides [see Fig. 1(a) for $j=1$ ]. The associated effective potential between first neighbors is therefore repulsive for equal-sided cells and attractive for cells with a different number of sides [see Fig. 1(b)]. This is the Aboav law [4] in the language of correlation. Increasing the topological distance $j$, the correlation and the potential shows oscillations that can be associated to the screening and antiscreening of the effective interaction.

In accordance with previous analyses on first-neighbors correlation $[13,16,17]$, we introduce the correlator $A_{j}(n, m)$ $\equiv\left[C_{j}(n, m) / p(n) p(m)\right]$, which is the probability to have an $n$-sided cell at a distance $j$ from an $m$-sided cell, given that the two cells exist [this correlator become approximately equal to the Boltzmann factor $e^{-\varphi_{j}(n, m)}$ as $s_{j}(n) \rightarrow p(n)$ for $j \rightarrow \infty]$. Figure 2 shows the plot of $A_{j}(n, m)$ vs $m$ for fixed $n=4, \ldots, 8, n \neq m$ and fixed $j=1,2,3$. There are theories $[7,18]$ that predict the bilinearity of $A_{1}(n, m)$ in $n, m$ using the maximum entropy argument. The poor fit to the straight line of the data in Fig. 2, and the strong deviation when $n$ $=m$, indicate that the bilinearity of $A_{j}(n, m)$ is a crude approximation. After all, this disagreement should not be too surprising as the potential defined in Eq. (5) manifests strong and long-range interaction present in soap froth. On the other hand, we find a surprisingly simple linear behavior of $A_{j}(n, n)$ vs $n$ for $j=1,2,3$. Figure 3 shows the plot of $A_{j}(n, n)$ vs $n$ for fixed $j=1,2,3$. The data can be described by the linear function $A_{j}(n, n)=\mu(j) n+\lambda(j)$ with coefficients $\mu(1)=0.193 \pm 0.008, \lambda(1)=-0.60 \pm 0.05 ; \quad \mu(2)$ $=0.121 \pm 0.005, \lambda(2)=0.36 \pm 0.03 ; \quad$ and $\quad \mu(3)=0.073$ $\pm 0.003, \lambda(3)=0.69 \pm 0.02$.

In conclusion, the data we present on soap froth strongly suggest that correlation effects exist and may be important in our understanding of the quasistatic and dynamics of froth. The data indicate that the correlation is within error bar nonzero up to the third shell at least, with a linear correlator for cells of the same number of edges, but nonbilinear behavior for cells of a different number of edges. Future theories of froth dynamics may want to consider these correlations. Although the nontrivial correlations beyond first shell and the linear law described in Fig. 3 are not well understood at this point, they may be important as the short-range interaction manifested by the Aboav-Weaire law and the observed longer-range interaction can compete with each other in the coarsening process of froth. We should also mention that 
different correlations are observed in the Voronoi network on random points, but no known explanation is available.

K.Y.S. acknowledges support from the Hong Kong Telecom Institute of Information Technology. T.A. acknowledges discussions with N. Rivier and D. Dubertret, support from the Hong Kong University of Science and Technology, and partial support from EU, HCM Program "Physics of Foams" under Grant No. CHRXCT940542 and by TMR Contract No. ERBFMBICT950380. W.Y.T. acknowledges support from Direct Allocation Grant No. 96/97.SC22 of the Hong Kong University of Science and Technology.
[1] J. Stavans, Rep. Prog. Phys. 56, 733 (1993).

[2] J. Stavans, E. Domany, and D. Mukamel, Europhys. Lett. 15, 479 (1991).

[3] H. Flyvbjerg, Phys. Rev. E 47, 4037 (1993); Physica A 194, 298 (1993).

[4] D. A. Aboav, Metallography 3, 383 (1974); 13, 43 (1980).

[5] D. Weaire, Metallography 7, 157 (1974).

[6] C. Godreche, I. Kostov, and I. Yekutieli, Phys. Rev. Lett. 69, 2674 (1992).

[7] N. Rivier, Philos. Mag. B 52, 795 (1985); Physica D 23, 129 (1986).

[8] M. Seul, N. Y. Morgan, and C. Sire, Phys. Rev. Lett. 73, 2284 (1994); C. Sire and M. Seul, J. Phys. I 5, 97 (1995).
[9] M. A. Fortes and P. Pina, Philos. Mag. B 67, 263 (1993).

[10] K. Y. Szeto and W. Y. Tam, Phys. Rev. E 53, 4213 (1996).

[11] T. Aste, K. Y. Szeto, and W. Y. Tam, Phys. Rev. E 54, 5482 (1996).

[12] T. Aste, D. Boose, and N. Rivier, Phys. Rev. E 53, 6181 (1996).

[13] G. Le Caer and R. Delannay, J. Phys. A 26, 3931 (1993).

[14] J. Stavans and J. A. Glazier, Phys. Rev. Lett. 62, 1318 (1989).

[15] K. Y. Szeto and W. Y. Tam, Physica A (to be published).

[16] M. A. Peshkin, K. J. Strandburg, and N. Rivier, Phys. Rev. Lett. 67, 1803 (1991).

[17] R. Delannay and G. Le Caër, Phys. Rev. Lett. 73, 1553 (1994).

[18] B. Dubertret, N. Rivier, and M. A. Peshkin (unpublished). 